\title{
Geometrical Effects in X-mode scattering
}

\author{
N. Bretz \\ Plasma Physics Laboratory, Princeton University \\ P. 0. Box 451, Princeton, New Jersey 08544
}

\section{ABSTRACT}

One technique to extend microwave scattering as a probe of long wavelength density fluctuations in magnet lcally confined plasmas is to consider the launching and scattering of extraordinary ( $X$-mode) waves nearly perpendicular to the field. When the incident frequency is less than the electron cyclotron frequency, this mode can penetrate beyond the ordinary mode culoff at the piasma frequency and avoid signiîicant distortions from density gradients typical of tokamak plasmas. In the more familiar case, where the incident and scattered waves are ordinary, the scattering is isotropic perperxdicular to the field. However, because the $x$-mode polarization depends on the frequency ratios and the ray angle to the magnetic field, the coupling between the incident and scattered waves is complicated. This geometrical form factor must be unfolded from the observed scattering in order to interpret the scattering due to density fluctuations alone. The geometrical factor is calculated here for the special case of scattering perpendicular to the magnetic field. For frequencies above the ordinary mode cutoff the scattering is relatively isotroplc, while below cutoff there are minima In the forward and backward directions which go to zero at approximately half the ordinary mode cutoff density.

\section{DISCLAIMER}

\begin{abstract}
This report was prepared as an account of work sponsured by an agency of the United States Government. Neither the United Stales Government nor any agency thereof, nor any of their employees, makes any warranly, express or implied, or assumes any legal liability or responsibility for the accuracy, completeness, or usefulness of any information, apparatus, product, or process disclosed, or represents that its use would not infringe privalely owned rights. ReTerence berein to any specilis commercial product, process, or service by trade name, Irademark, manufacturer, or otherwise does not necessarily constitule or imply its endorsement, recommendiation. or favoring by the United States Governnenl or any agency thereof. The views and opinions of authors expressed herein do not neechsuriiy state or reflecl those of the United States Government or any agency thercol.
\end{abstract}




\section{INTRODUCTION}

The scattering of microwaves by density fluctuations is usually carried out by launching and scattering ordinary mode radiation. 1,2 When this is done in the plane perpendicular to the magnetic field, the scattered radiation pattern of a single electron is that of a simple dipole oriented along the magnetic field and is, thus, isotropic in the scattering plane. This simplifies the interpretation of the scattering due to electron density fluctuations. Density correlations at different values of $k\left(=\left|k_{i}-k_{5}\right|\right)$ determined by the incident (i) and scattered (s) wave vectors only need to be normalized to the relative gain of the launching and receiving anternae. However, much of the interesting phenomena in tokamak plasmas takes place at relatively long wavelengths which can be observed only at small scattering angles (at correspondingly poor spatial resolutien) or by.using long probe wavelengths. For example, in tokamaks drift waves which most affect transport occur at $k p_{s} \approx 0.3$ where $p_{s}=\sqrt{T_{\mathrm{g}}} / \bar{M}_{j} / t_{c i}$. Thus, for a deuterium plasma at a toroidal field $B_{T}$ of $5 T$ and $T_{e}=2 \mathrm{keV}$, the relevant fluctuation wavelengths have $\lambda=2 \pi / k \approx 1.5 \mathrm{~cm}$. The scattering condition, $k=4 \pi[\sin \theta / 2] / \lambda_{j}$, where $\theta$ is the angle between $k_{j}$ and $k_{5}$, implies that probe wavelengths on the order of $1 \mathrm{~cm}$ are needed if good spatial resolution is required. For the ordinary mode the wavelength limit is set by a cutoff at the plasma frequency and refraction near the cutoff from density gradients which causes distortion of the ray paths. The density which cuts of $30 \mathrm{GHz}(1 \mathrm{~cm})$ radiation is $1.1 \times 10^{19} \mathrm{~m}^{-3}$; 50 the tokamak core is not accessible at the wavelengths of interest.

One way arotnd the timitation imposed by the ordinary $(0)$ mode cutoff is to consider launching and receiving the extraordinary $(X)$ mode. For perpendicular propagation and when the incident wave frequency $\left(f_{j}\right)$ is below the electron cyclotron frequency $\left(r_{C}\right)$, the $x$-mode cut-orf condition is $f_{i}{ }^{2}+f_{i} f_{c}-f_{p}{ }^{2}=0$ giving a substantially higher cut-off density. In the prevtous example when the incident frequency is $30 \mathrm{GHz}$ and $B_{T}=5.0 \mathrm{~T}$, then $f_{c} / f_{i}=4.7$, and the $x$-mode cutorf occurs at 
$6.3 \times 10^{19} \mathrm{~m}^{-3}$

However, the $X$-mode is elliptically polarized perpendicular to the magnetic field, and the ellipticity depends on the ratios $f_{c} / f_{j}, f_{p} / f_{i}$, and on the ray angle to the magnetic field. As a result, the coupling between the incident and scattered waves is complicated, and the isotropic scattering form factor for perpendicular scattering characteristic of the 0-mode must be replaced by a factor appropriate for the $\mathrm{X}$-mode. The purpose of this paper is to calculate this factor arxd to examine whether it detracts from the value of using $X$-mode scattering to examine density fluctuations.

It will be shown that, for the special case of scattering nearly perpendicular to the magnetic field, the geometrical form factor is relatively isatropic at densities above the D-mode cutoff, but for lower densities the form factor has minima in the forward and backward directions. When $f_{c} / f_{i}>1$, these minima go to zero at about half the 0 -mode cut-off density. At all angles the cross section is reduced by approximately $f_{c} / 2 f_{j}$.

The general form of the scattering cross section for a plasma in the presence of a magnetic field, including the geometrical form factor, has been worked out by Akhiezer et at..$^{3-5}$ and by Simonich. 6,7 The two formulations appear somewhat different but agree on the basic coupling term. The essential difference between the two calculations is due to an extra factor in simonich's expression which accounts for the difference between the phase and group directions and for the curvature of the index of refraction surface. The physical problem considered here requires that all features be included in the final result.

\section{FORMULATION}

The scattering geometry is shown in figure 1a. The angles of the incident and scattered propagation vector directions $\boldsymbol{k}_{j}$ and $k_{s}$ to the magnetic field $\mathrm{B}$ are $\theta_{j}$ and $\theta_{\mathbf{S}}$. The magnetic rield will be assumed to lie in the $z$ direction and $k_{\mathrm{j}}$ to lie in the $x-z$ plane. The argle between 
the $\mathbf{B}, \mathbf{k}_{\mathbf{i}}$ plane and the $\mathbf{B}, \mathbf{k}_{\mathbf{S}}$ plane is $\Phi$. Characteristic plane waves in a homogeneous cold plasma are described by the equation

$$
k x(k \times E)-\omega^{2} / c^{2} \overline{\bar{\varepsilon}} \cdot E=0 \text {. }
$$

where if $k$ lies in the $x-z$ plane,

$$
\overline{\bar{\varepsilon}}=\left[\begin{array}{ccc}
S & -i D & 0 \\
i D & S & 0 \\
0 & 0 & P
\end{array}\right]
$$

and in the electron approximation $\left(M_{j} / M_{e} \gg 1\right)$

$$
\begin{aligned}
& S=1-\alpha /(1-\beta) \\
& D=-\beta^{1 / 2} \alpha /(1-\beta) \\
& P=1-\alpha,
\end{aligned}
$$

where

$$
\alpha=f_{p}^{2 / f^{2}}, \quad \beta=f_{c}^{2 / f^{2}} .
$$

Solutions exist when ||$k x k x-\omega^{2} / c^{2} \overline{\bar{\varepsilon}}||=0$ or when

$$
\left.k^{2} c^{2} / \omega^{2} \equiv n^{2}=1-\alpha / 1-\beta \cos \theta\left[G \pm\left(\sigma^{2}+1\right)^{1 / 2}\right\}\right] \text {. }
$$

where

$$
G=\frac{\sin ^{2} \theta}{2(1-\alpha) \cos \theta}
$$

The lower sign corresponds to the 0-mode and the upper sign to the $x$-mode. Thus, for 0 -mode perpendicular propagation

$$
n^{2}=P=1-\alpha
$$

and

$$
E=(0,0,1) .
$$

For the $x$-mode

$$
n^{2}=\left(S^{2}-D^{2}\right) / S=1-\alpha(1-\alpha) /(1-\alpha-\beta)
$$




$$
E=(1, \mathrm{i} 0 / \mathrm{S}, 0)
$$

These are the two characteristic modes which propagate in the direction perperdicular to $\mathbf{B}$. Cutorf is characterized by $n=0$ and resonance by $n->\infty$. The polarization of the 0 -mode is linear parallel to $B$ while the $X$-mode is elliptically polar ized in the plane perpendicular to $\mathbf{B}$.

\section{SCATTERING CROSS SECTION}

The scattering cross section has been calculated assuming that the ooserver is far from the scattering volume (Born approximation), that there are a large number of fluctuation wavelengths in the scattering volume, and that the scattering or absorption mean-free path is long compared to the plasma dimensions. The notation of Akhiezer et al for the scattering cross section will be used with appropr iate modifications for the index of refraction curvature and phase versus group factors. The differential cross section is

$$
d \sigma=r_{0}^{2} \Gamma<\delta n_{e}^{2>} k, f d \Omega d f \text {, }
$$

where $\Gamma=\left[f_{i}{ }^{2} f_{s}{ }^{2} / f_{p}{ }^{4}\right] \mathrm{H}|\xi|{ }^{2} \mathrm{H} /|\mathrm{C}|$ is the geometrical form ractor. $r_{0}$ is the classical electron radius,

$\left\langle\delta n_{e}{ }^{2}\right\rangle_{k, f}$ is the ensemble-averaged, Fourier-transformed, density autocorrelation function,

$d \Omega$ is the solid angle sampled by the detector, and $d f$ is the frequency interval sampleci by the detector.

The terms in $\bar{\Gamma}$ are identified as follows

$$
\begin{aligned}
& R=n_{s}{ }^{2 / n_{j}}\left(\left|E_{i}\right|^{2}-\left|E_{i} \cdot k_{i}\right|^{2 / k_{j}}\right) \Sigma_{s} E_{s}^{*} \cdot \bar{\varepsilon}_{5} \cdot E_{s} \\
& \xi=\Sigma_{5} E_{s}{ }^{k} \cdot\left(\bar{\varepsilon}_{s}-1\right) \cdot E_{i} \\
& H=\cos \delta_{s} \cos \delta_{i} \\
& C=\left[1+\sin ^{2} \delta_{s}-\left(n_{s} \cdot n_{s}\right) \cos ^{2} \delta_{s}\right] \sin p_{s} \cos \delta_{s} / \sin \theta_{s}
\end{aligned}
$$




$$
\begin{aligned}
& \tan \delta_{s}=-n_{s} / n_{s} \\
& \delta_{s}=p_{s}-\theta_{s} \\
& n_{s} \equiv d n_{s} / d \theta_{s} \\
& n_{s}=d^{2} n_{s} / d \theta_{s}{ }^{2}
\end{aligned}
$$

and 1 is the unit tensor.

The matrices in $\mathrm{R}$ and $\xi$ must be summed over both of the final state normal modes ( $O$ and $X$ ) and, of course, the initial polarization may itself be a sum over any linear combination of normal modes for the incident direction and frequency. The term $\mathrm{H}$ describes the correction due to the difference in the phase and group velocities, and $C$ is the curvature oi the index of refracijon surface. The angle between the group velocity and the field direction is $p$, and $\delta$ is the angle between the phase and group directions as showr in figure it. For high frequencies the term $\mathrm{H} /|\mathrm{C}|$ reduces to unlty and the term $\mathrm{R} \mid \mathrm{q}^{2}$ becomes the familiar argular pattern of a simple dipole. The main coupling term $R|\xi|^{2}$ is common to both the Akhiezer and Simonich ${ }^{8}$ calculations while $H /|C|$ describes the group versus phase and index curvature components introduced by simonich.

For the coordinate system which has been chosen here the scattereu wave does not iie in the $x-z$ plane and must be represented as a ratation on the simpler form in which the normal mode has been expresseo. For example, when one considers $X$-mode scattering and $\theta_{i}=\theta_{s}=\pi / 2$, the incident wave $E_{i}=\left(1, i D_{i} / S_{i}, 0\right)$ whereas the scattered field is

$$
E_{s}=\left[\begin{array}{ccc}
\cos \phi & -\sin \phi & 0 \\
\sin \phi & \cos \phi & 0 \\
0 & 0 & 1
\end{array}\right]\left[\begin{array}{c}
1 \\
\mathrm{iD}_{\mathrm{s}} / \mathrm{s}_{\mathrm{s}} \\
0
\end{array}\right]
$$

The term containing $\mathrm{E}_{\overline{5}}{ }^{*} \cdot(\overline{\bar{E}}-1) \cdot \mathrm{E}_{\mathbf{i}}$ contains the primary angular dependerce for the form factor, and can go to zero for forward and backward scattering $\left(E_{\mathbf{s}}= \pm E_{j}\right)$. Physically this means that the rate of change of the index of refraction with density is zero at some density. 
An examination of the index shows that this must occur on the interval $0<\alpha<1$ for $\beta>1$ since $n^{2}=1$ at $\alpha=0$ and 1 , and $n^{2}>1$ in between.

A5 given, the cross section expresses the coupling between arbitrary incident and scattered waves. An expression for the general case is possible but is sufficiently complex that important effects would not be revealed easily. The case of interest here is $X$-mode incident where $\theta_{j}=\theta_{5}=\pi / 2$ and $f_{5}=f_{j}$. It is expected on physical grounds that there will be no 0 -mode wave scattered since there is no component of the polarization field in the incident wave which is parallel to $B$. In fact, $\xi=0$ for the matrix element corresponding to scattering from $X$ to 0 -mode. It will be sufficient to focus attention only on the scattered $X$-mode. This leads to significant simplifications in the general expression. First, the angle $\delta$ between the phase and group velocities goes to zero, and the curvature term $\mathrm{H} /|\mathrm{C}|$ becomes $\left|2-n^{2}\right|$. Furthermore, the conventional scattering angle, $\theta$, between the wavevectors $k_{i}$ and $k_{s}$ becomes the angle $\boldsymbol{\phi}$. In this case, after some a!gebra

$$
\begin{aligned}
& E_{S}{ }^{*} \cdot \varepsilon_{s} \cdot E_{S}=S\left(S^{2}-D^{2}\right) / D^{2} \\
& \left|E_{i}\right|^{2}-\left|E_{i} \cdot k_{i}\right|^{2 / k_{i}{ }^{2}=s^{2} / 0^{2}} \\
& |\xi|^{z}=\left\{\left[D\left(S^{2}-D^{2}\right)-2 S D\right]^{2} \sin ^{2} \theta\right. \\
& \left.+\left[S\left(s^{2}-D^{2}\right)-\left(S^{2}+D^{2}\right)\right]^{2} \cos ^{2} \theta\right] / D^{4} \\
& \text { and } \\
& H /|C|=\left|1-n_{s} \cdot 1 / n_{S}\right|=\left|2-n^{2}\right| .
\end{aligned}
$$

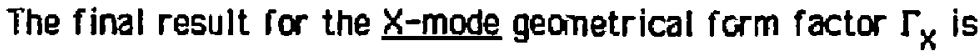

$$
\Gamma_{x}=\frac{\beta\left[1-\alpha^{2}-\beta\right]^{2} \sin ^{2} \theta+\mid\left(\gamma^{2}-\beta(\beta-1)\right]^{2} \cos ^{2} \theta}{\left|\gamma^{3}\left(1-\alpha^{2}-\beta\right)\right|},
$$

where $\gamma=1-\alpha-\beta$. The form factor is seen to be symmetric about $\Theta=\pi / 2$, and in the for'ward and backward directions the cross section goes to zero when the condition 


$$
\alpha=\sqrt{\beta(\beta-1)}-(\beta-i)
$$

is met. In the example used above $\beta=(4.7)^{2}$, and $\Gamma_{x}=0$ at $\alpha=0.49$ which corresponds to a density of about half the 0-mode cutoff. This is the same condition which is obtained by setting $d n^{2} / \mathrm{dn}_{\mathrm{e}}$ or $\mathrm{dn} \mathrm{n}^{2} / \mathrm{d} \alpha=0$. At the 0 -mode cutorf $(\alpha=1)$ the form factor is simply

$$
\Gamma_{X}=\left(1 / \beta^{2}\right) \sin ^{2} \theta+(1 / \beta) \cos ^{2} \theta
$$

And when the density reaches the $x$-mode cutoff $\left(\alpha=\beta^{1 / 2}+1\right), \Gamma_{X}$ becomes isotropic and $I_{X}=2 /\left(\beta^{1 / 2}+1\right)^{2}$. This occurs because the $X$-mode polarization becomes circular at cutoff, and the scattered polarization fields are equally matched in all directions. Figures $2 a$ and $2 b$ show $\Gamma$ as a function of $\theta$ for various values of $\alpha$ for our example $\beta=(4.7)^{2}$ and ior $\beta=4.0$. From a practical viewpoint the cross section does not change so rapidly for the most interesting values of $\alpha$ and $\beta$ that unfolding $\left\langle\delta n_{e}{ }^{2\rangle}\right.$ presents large difficulties. However, the cross

section is reduced approximately proportional to $\lambda^{-1}$ which compensates for an increase in the cross section proportional to $\lambda^{2}$ that might be expected to come from $\left\langle\delta n_{e}{ }^{2\rangle}\right.$ for conerent waves.

The corresponding result for the 0 -mode incident and scattered at $\theta_{i}=\theta_{S}=\pi / 2$ gives

$$
\Gamma_{0}=1 /(1-\alpha),
$$

showing the expected isotropy and resonance at $\alpha=1$. In the high frequency limit $(\alpha=\beta=0), \Gamma_{X}=\cos ^{2} \theta$ and $\Gamma_{0}=1$ which is the emission pattern of a simple dipole.

\section{DISCLSSION}

In order to apply the idealized calculation given here to the more complex situation in a tokamak, one must know how characteristic modes propagate nearly perpendicular through sheared rields, through 
density gradients, and in a hot plasma. Fortunately these complications do not change the result significantly. It can be shown that away from resonances and cut of $f$ the polarization fielos of the raly remain essentially those given by the cold plasma relations, and the ray propagates in such a way that the $x(0)$-mode fields remain perpendicular (parallel) to the local inagnetic field. In the case of magnetic fields sheared perpendicular to the ray direction Boyd ${ }^{9}$ has shown that when the density is sufficiently high, the modes propagate so that the polarization plane rotates with the local magnetic field. For shears typical of a tokamak and for the conditions in our example, the critical density is $\mathrm{n}_{\mathrm{e}} \approx 10^{14} \mathrm{~m}^{-3}$. Thus, in all but a very small region at the edge the mode propagates in a very simple way. Several simulations using modeled tokamak fields and densities have been treated with a rats tracing code ${ }^{10}$ and give similar results. That is, an $X$-mode launched at the edge propagates into the plasma and refracts due to density gradients, but its polarization field remains perpendicular to the local magnetic field. For propagation away from cutoffs and resolances the cold plasma approximation is adequate in describing the ray paths. This means that the scattering cross-section calculated here for pure $X$-mode scattering is appropriate as long as the coordinate system defined by $\mathbf{B}$ is taken locally. Ray tracing can be used to infer the local scattering angles and the location of the scattering region as long as the density and temperature (and, therefore, B) profiles are known. For many cases of practical interest, and when refraction effects are not too severe, this can be done with sufficient accuracy.

In practice, the choice of $\lambda_{i}$ is influenced not only by a desire to observe long wavelength fluctuations consistent with various access and cut-off phenomena, but by plasma emission which can mask the scattered signals near the plasma frequency and harmonics of the electron cyclotron frequency. ${ }^{11,12}$ Minima between these emission peaks are good regions to do scattering. However, when there is a small population of runaway electrons, these gaps fill in and there can De significant emission near the plasma frequency. 


\section{CONCLIJSION}

A general formula is given for the angular dependence of the scattering cross section due to the coupling of polarization fields in an anisotrcpic plasma for the special case in which the incident and scattered waves are $x$-mocie propagating perpendicular to the magnetic field. This form factor has been shown to be symmetric about $\Theta=\pi / 2$ and to exhibit minima in the forward and backward directions. When $\beta=f_{C} / f_{i}>1$, there is a density less than the 0-mode cut-of $f$ density for which the minima go to zero. For $\beta>2$ this density is approximately half the cut-cff density. Above the 0-mode cutoff the form factor becomes isatropic and has a value of order $2 / \beta$. This result may be used to unfold the scattering due to density fluctuations from observations of the scattering of $x$-mode radiation in the plane pependicular to the toroidal field of a tokamak plasma. Finally, the results given here show that the two previous treatments of the problem are consistent, but that the work of simonich, in addition to treating the ray problem, includes signif icant effects due to the difference between the ray and group velocities and due to the curvature of the index of refraction surface.

\section{ACKNOWLEDGMENTS}

The author wishes to thank P. Efthimion, A. Kritz, D. Boyd, F. Perkins, and $E$. Mazzucato for their comments and encouragement in the course of this work.

This work was supported by the United States Department of Energy Contract No. DE-ACO2-76-CHO-3073. 


\section{REFERENCES}

1. Mazzucato, F., Phys. Fev. Lett. 48 (1982) 1828.

2. Brower, D. L., Feetles, W. A., Lurmann, Jr, N. C., and Savage, Jr. R. L., Phys. Rev. Lait. 54 (i985) 689.

3. Akhiezer, A. I., Prokhoda, I. G., and Sitenko, A. G., \& $\therefore$ Phys. - JETP 6 (1958)576

4. Akhiezer, A. I, Akhiezer, I. A., and Sitenko, A. G., Sov. Phys. - JETP 14 (1962) 462.

5. Akhiezer, A. I., Akhiezer, I. A., Polovin, R.V., Sitenko, A. G., and Stepanov, K. N. Coliective Oscillations in a Plasma. Pergamon Press (1967) Ch. V.

6. Simonich, D. M., Radio Wave Scattering from Random Fluctuations in an Anisotropic Medium Ph.D. T̈hesis, University of Illinois (1971).

7. Simonich, D. M., and Yeh, K. C., Radio Sci. 7 (1972) 29 I.

8. Simonich uses the normalization $E^{\star}-\varepsilon-E=\dagger$ whereas the expression used by Akniezer (and adopted here) is independent of the field normatization.

9. Bojd, D. A., Proc. 5th Int1. Workshop on Electron Cyclotion Emission and Electron Cyclotron Heating, 1985, San Diego, GA Technologies Report GA-A13294.

10. Kritz, A. H., Hsuan, H., Goldfinger, R. C., Batchelor, D. B., Proc. of the 3rd Joint Varenna-Grenoble Intl. Sym., Grenoble (!982).

11. Costley, A. E. and TFR Group, Phys. Rev. Lett. 38 (1977) 1477.

12. Hutchinson, 1. H. and Komm, D. S., Nucl. Fusion 17 (1977) 1077. 


\section{FIGURE CAPTIONS}

FIG 1. The relationship between the incident and scattered rays and the magnetic field is shown in la. The relationship between the phase and group velocities and the magnetic field is shown in $t h$.

FIG 2. The geometrical form factor $\Gamma$ versus the scattering angle $\Theta$ is shown in $2 a$ for the case $\beta=(4.7)^{2}$ ard in $2 b$ for $\beta=4$ for several values of the parameter $\alpha$. 
(a)
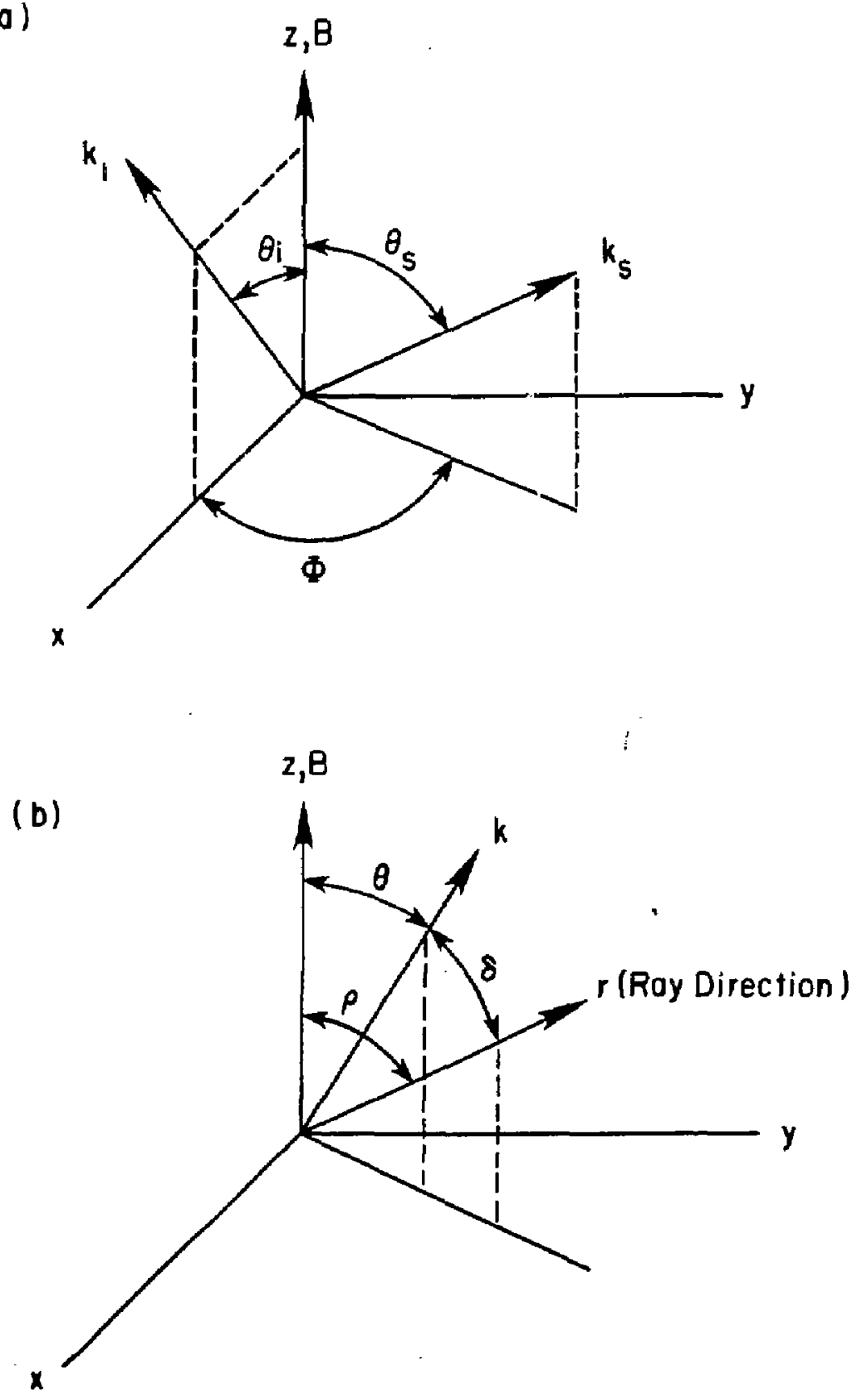

Eig. 1 

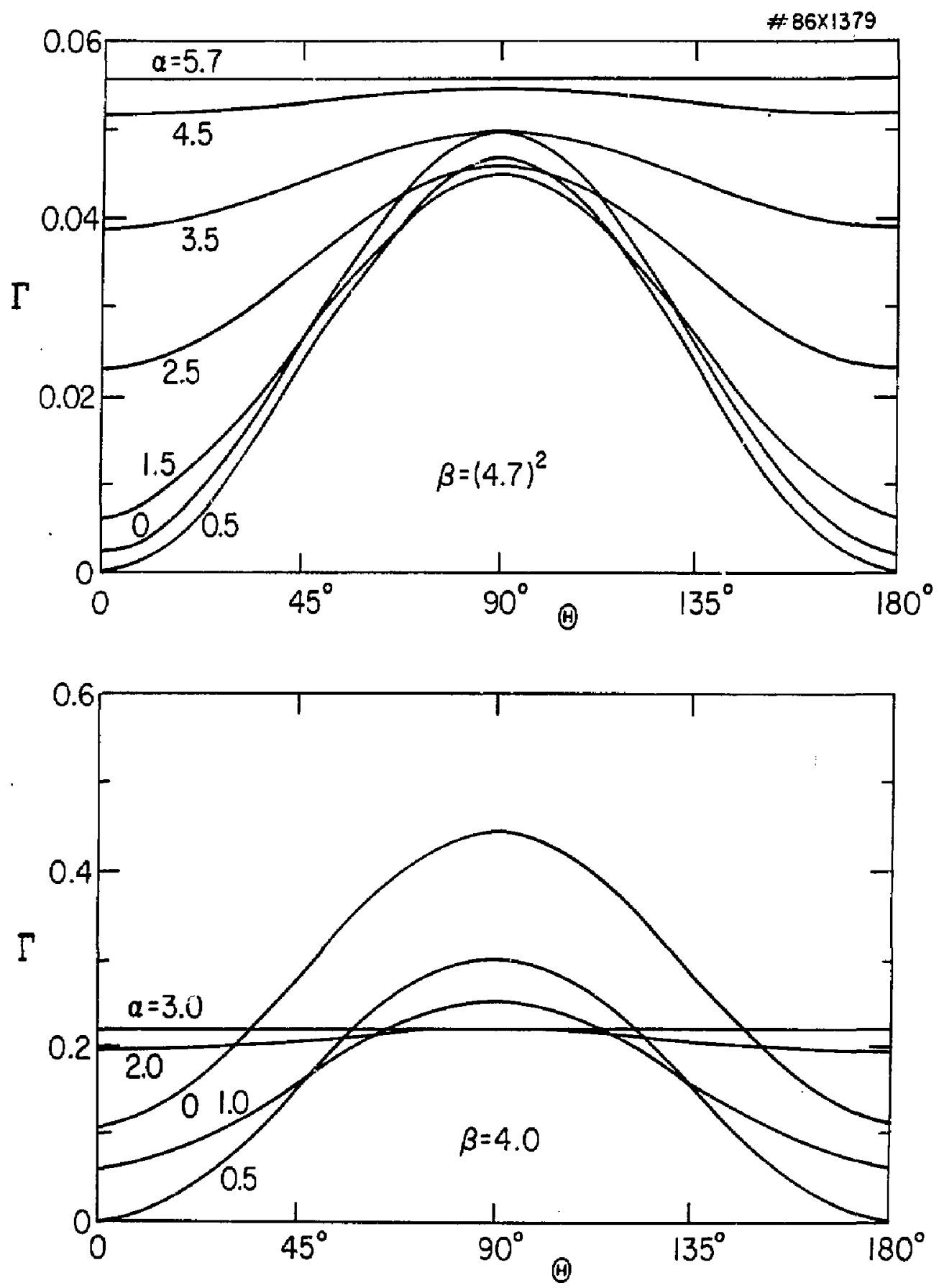

Fig. 2 
Flasina Res Lab, Austra Nat'l Univ, Austaklia

Jr. Frank J. Paolonl, Unle ot wollongong, AUSTRALIA

Prof. 1.R. Jonos, Flinders Univ.., AUSTRALiA

Prof. M.H. Grannan, Univ SyGitay, MUSTRaLIA

Prof. F. Cad, Inst Theo Phys, Austria

M. Goossens, Astronomisch instituut, BELGILM

Prot. R. Bousijug, Laboratorlum vacr Natuurkunda, BELGIUM

Or. D. Palumbo, Dg XII Fuslon Prog, BELGIUM

Ecole Rcyale MIlltaira, Lob de Phys Plasmas, BELGily

Dr. P.H. Sakanaka, Un 1v Estadual, BRAZIL

LIb, \& Doc. DIv.. Instltuto de Pesqulsas Espaclala, RRNIL

Jr. C.R. James, Unlv of Alberta, CANADA

Prof. J. Telchmann, Univ of Montreal, CANMDA

Or. H.M. Skarsgard, Unlv of Saskatchevan, CANADA

Prot. S.R. Sraenivisan. Untuersity of Calgory, Canada

Prot. Tudor H. Johnstan, INRS-Energle, CANADA

Or. Hannes aarnard, Univ aritish Columbla, Canada

Dr. M.P. Bachynskl, MPB Technologios, Inc., CANADA

Chalk RIver, Nucl Lab, CANADA

Z rengw LI, SW Inst Physles, CHINA

Library, Tsing Hua Unluarsity, CHINA

LIorarlan, Institute of Physies, CHiNA

Inst Plasm Phy5, Acadenla Sinlca, CHINA

Or. Peter Lukz=, Konenskeho Univ, CZECHOSLOVAKIA

The Librartian, Culham Laboratory, ENGLAND

Prof. Schatzman, Observatolre de NICe, FRARCE

J. Radet, CEN-BPG, FRANCE

JET Reading Room, JET Jotnt Undertaking, ENGLAND

AM Dupas LIbrary, AM Dupas Ll brary. FRANCE

Dr. Tom Hual, Academy Blbllographlc, HONG KONG

Preprint Library, Cent Res Inst Phys, HUNGary

Or. R.K. Canajianl, VIkram Univ. INDIA

Dr. B. Dasgupta, Saha inst, INDIA

Or. P. Kaw, Physlcal Research Lab, INDIA

Dr. Phtllip Rosanau, Israal Inst' Tach, ISFAEL

Prof. 5. Cuparman, Tol Aviv Unlverslty, ISRAEL

Prot. G. Aostagni. UnIv DI Padova, ITALY

LIbrarlan, Int'l Ctr Theo Phys, ItALY

Miss Clella De Palo, Assoc EURATOM-ENEA, ITALY

BIbllotece, del CNR EURATOM, ITALY

Dr. H. Yanoto, Toshlba Ros \& Dav, JAPAN

Diroc, Dept. Lg. Tokanak Dev, JAERI, JAPAN

Prot. Nobuyuál Inoue, UnIversity of Tokyo, JAPAN

Resesrch Info Center, Nagoya Unlverslty, JAPAN

Prot. Kyoll NIshlkawa, Univ of HIroshima, JAPAN

Prof. Sigeru Morl, JAERI, JAPAN

Prof. S, Tanaka, Kycto UnIyorsity, JAPAN

Library, Kyoto Unluors Ity. JAPAN

Prot. I chtro Kawakant, Nthon UnIv, JAPAN

Prof. Sotoshl Itoh, Kyushu University, JAPAN

Dr. D. I, Chal, Adu, Inst Sel \& Tech, KOREA

Tech In fo DIylsion, KAERI, KOREA al ol lathesk, Fom-Inst Vaor PI asma, NETHERLANOS

arof, E.S. Llley. University of Wolkato, NEW ZEALANO

Prot. J.A.C. CEbral, Inst Suderlor TECn, PORTUGAL

Or. Octavlan Petrus, ALI CLRA Unlversity, ROMANia

Prof. M.A. Helluerg, University of Natal, SO AfRICA

Dr. Johom de Villlers, Plasera Physies, Nucor, SO AFRICA

Fuston DIV. LIorary, JEN, SPAIN

Prof. Hans Wilhelmson, Chalmars UnIr Tech, SWEDEA

Or. Lonnart Stentlo, University of LMEA, SWEDEN

LIbrary, Royal Inst Tach, SWEDEN

Centre de Recherchesen, Ecole Polytach Fed, SWITZERLAND

Dr. V.T. Tolak, Kharkoy Phys Tech ins: USSR

Dr. D.D. Ryutov, \$lberian Acad Se1, US\$R

Dr. G.A. Eliseov, Kurchatov Instituta, USSR

Dr. $V_{*} A, G$ lukhikh, Inst Electrothysleal, USSR

Institute Gen, Fhysles, USSR

Prof. T.S.M. Boyd, Unlv College N wales, waLES

Dr. K. Senlndier, Gunr Universitot, W. GERMANY

ASDEX Reading Rm, IPPMaxtPlanck-Institut fur

Plasmaphysjk, F,R.G.

Nuclaar Res Estab, Julleh Ltd, W. GERMANY

Librarlon, Max-Planck Institut, w. GERMANY

Glbllothek, Inst Plasmaforschung, W. GERMANY

Prof, R.K. Jonev, Inst Phys, YugOSLAVIA 